\title{
Local Electric In-Plane Potential Fluctuations in the CdTe/CdMgTe Based Multiple Quantum Wells
}

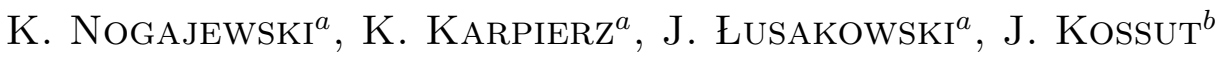 \\ AND M. GRYNBERG ${ }^{a}$ \\ ${ }^{a}$ Institute of Experimental Physics, University of Warsaw \\ Hoża 69, 00-681 Warsaw, Poland \\ ${ }^{b}$ Institute of Physics, Polish Academy of Sciences \\ al. Lotników 32/46, 02-668 Warsaw, Poland
}

\begin{abstract}
Unusual features in the magnetophotoconductivity spectra registered under far infrared illumination of the $\mathrm{CdTe} / \mathrm{CdMgTe}$ based multiple quantum wells, uniformly $n$-doped are presented. It is shown that each spectrum exhibits one or two peaks of non-symmetrical shape, with position of their maxima dependent on the voltage applied to the sample. The peaks, observed in the configuration of the crossed electric and magnetic fields, are strongly shifted by a relatively weak in-plane electric field - of the order of $10-50 \mathrm{~V} / \mathrm{cm}$. Two different approaches to explain the observed influence are presented. Both are based on a two-step process leading to the photoconductivity signal. The first approach assumes that only the process of photon absorption is influenced by the external fields, the second one assumes that only the process of phonon assisted electron transfer from the excited donor state into the conduction band is influenced by the external fields.
\end{abstract}

PACS numbers: 71.55.Gs, 72.15.Rn, 72.80.Ey, 73.21.Fg

\section{Introduction}

Local electrostatic potential fluctuations exist in most of structures of reduced dimensionality, which are extremely important objects of interest of many research groups. The fluctuations originate mainly from surface and/or interface states, ionized centers and dislocations propagating through the crystal lattice. Usually, their presence is considered as a complication in the experimental work, because of decreasing the sensitivity of applied techniques or a broadening of spectral lines in spectroscopic measurements. An example of such spectroscopy experiment is magnetospectroscopy of shallow donors [1-3] where one gets spectral lines 
by applying the external magnetic field and splitting energy levels of a single center. The half width of such lines is direct evidence and a measure of local electric fields and their gradients [4]. By applying this technique one can determine, in the extremely precise way, even the existence of weak local electric fields and their inhomogeneities. This allows to draw conclusions about the quality of the sample and the degree of internal disorder.

In some cases, however, local fluctuations of the electrostatic potential are a desirable feature which enables observation of new phenomena. In the $\mathrm{CdTe} / \mathrm{CdMgTe}$ based quantum well (QW) sample the presence of electrostatic disorder allowed to observe unexpected phenomena - the same position of maxima in the magnetophotoconductivity spectra regardless of the incident photon energy [2]. What is more, the position of maxima is sensitive to the bias voltage applied in the plane of the QW [5].

The goal of this paper is to show that the same behavior and the sensitivity to the in-plane voltage takes place also in the structure consisting of many QWs and to understand possible mechanisms responsible for the origin of the observed maxima in the magnetophotoconductivity spectra. These mechanisms should take into account the up-to-date model, based on the existence in the structure of so-called mixed quantum dots (MQD), which are originating from an additional in-plane electron confinement due to local potential fluctuations together with the potential of the quantum well barriers [5].

\section{Samples and experiment}

Experiments were carried out on the sample whose active part consisted of 20 $\mathrm{CdTe} / \mathrm{Cd}_{0.8} \mathrm{Mg}_{0.2} \mathrm{Te}$ quantum wells, each one $16 \mathrm{~nm}$ wide and $240 \mathrm{meV}$ deep in the conduction band, separated by $48 \mathrm{~nm}$ thick barriers. Both: barriers and quantum wells were doped uniformly with iodine donors at the level of $\approx 2 \times 10^{16} \mathrm{~cm}^{-3}$. The sample was a rectangular slab with the dimensions $\approx 3 \mathrm{~mm} \times 4 \mathrm{~mm}$, grown by the molecular beam epitaxy method on a semi-insulating GaAs substrate with a $7 \mu \mathrm{m}$ thick buffer layer of undoped $\mathrm{CdTe}$, followed by a $0.5 \mu \mathrm{m}$ undoped layer of the barrier material and then by $0.5 \mu \mathrm{m}$ layer of the barrier doped by iodine. The top layer was a $48 \mathrm{~nm}$ thick barrier. The separation between the quantum wells was big enough to allow us to treat them as separate quantum wells. The magnetophotoconductivity spectra due to far infrared (FIR) illumination as a function of an external magnetic field have been measured in the magnetic fields up to $6 \mathrm{~T}$. The source of FIR monochromatic light with the photon energies of $10.4 \mathrm{meV}$ or $12.8 \mathrm{meV}$ was a FIR molecular laser, optically pumped by a $\mathrm{CO}_{2}$ laser. The measurements were done at the temperature $4.4 \mathrm{~K}$, for several values of the bias voltage $U_{\mathrm{b}}$ - ranging from $4 \mathrm{~V}$ to $22 \mathrm{~V}$. The inset in Fig. 1 shows the scheme of applying voltage to the sample. The photocurrent (due to chopped FIR illumination) was measured as a voltage drop on the load resistor $R_{\mathrm{L}}$ with a lock-in technique. During experiments the sample was permanently illuminated 
with the visible light coming from a monochromator, with the photon energy $h \nu=$ $2.1 \mathrm{eV}$. Its role was to deliver electrons to the electric levels in the quantum wells and to populate shallow donor states in order to make conductivity experiments possible. It is worth to notice that without this illumination the sample resistance exceeded $10^{11} \Omega$.

\section{Results}

Figure 1 shows a set of spectra obtained for different external in-plane bias voltages. For all of them we notice the characteristic peak, at $\approx 3.7 \mathrm{~T}$. This is a kind of a "marker" originating from the $1 s-2 p_{+}$transitions in the GaAs substrate donors. It proves that the FIR light and visible light penetrate the whole active part of the sample reaching the substrate. A more interesting feature the maxima originating from $\mathrm{CdTe} / \mathrm{CdMgTe}$ quantum wells - are marked by the vertical arrows on each spectrum. This feature, named as "A", exhibits a very strong sensitivity to the applied in-plane voltage. For $U_{\mathrm{b}}=4 \mathrm{~V}$, the maximum of "A" is placed at $\approx 1 \mathrm{~T}$, while for $U_{\mathrm{b}}=18 \mathrm{~V}$ it shifts almost up to $\approx 6 \mathrm{~T}$. A similar behavior was observed previously for the sample with a single QW [5], but in this case the applied voltages (for the same dimensions of the sample) had to be changing between $10 \mathrm{~V}$ and $40 \mathrm{~V}$, and the observed magnetic field shift was from 1.5 to $3 \mathrm{~T}$. We have also checked the shape of the multiple QW sample magnetophotoconductivity spectra for FIR photon energy equal to $12.8 \mathrm{meV}$. Similarly to the single QW $[1,2,5]$, the position of the feature "A" in this sample is the same for both FIR photon energies.

The last observable in the spectra recorded for multiple quantum well sample is the peak marked by the oval in Fig. 1 named "B". This peak becomes visible for spectra taken for $U_{\mathrm{b}}>12 \mathrm{~V}$. Its position changes very weakly with increasing voltage - the magnetic field shift is equal to $0.4 \mathrm{~T}$ between $U_{\mathrm{b}}=12 \mathrm{~V}$ and $22 \mathrm{~V}$.

\section{Interpretation}

Previous experiments done on a single $\mathrm{CdTe} / \mathrm{Cd}_{0.8} \mathrm{Mg}_{0.2} \mathrm{Te}$ quantum well $[1,2,5]$ led to the conclusion that the observed peaks in the magnetophotoconductivity spectra are due to the FIR light absorption by electrons transferred between the states of shallow donors split by the external magnetic field [1]. The origin of a photoconductivity $\mathrm{PC}(B)$ signal in magnetophotoconductivity experiments is always a two-step process of (i) absorption of a FIR photon by an electron followed by (ii) the transfer of the excited electron into the conduction band, mainly by the Lifshitz mechanism [6]. External electric and magnetic fields may influence each of these steps. Additionally, for both kinds of samples, either a single or multiple QWs, one expects strong local spatial changes of electric potential potential fluctuations [1]. The sources of them are mainly uncompensated surface states and ionized donors as well as charged states which accompany the dislocations. Because of a much wider extent of the structure along the $z$ direction in 


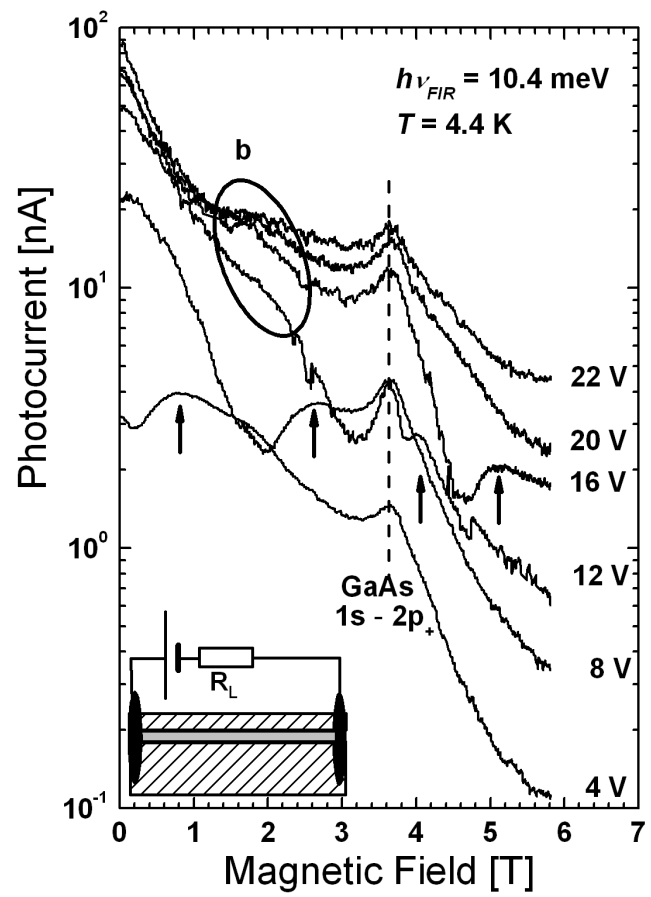

Fig. 1. Photoconductivity spectra due to FIR illumination with photon energy $h \nu=$ $10.4 \mathrm{meV}$, taken at $T=4.4 \mathrm{~K}$. Each spectrum is labelled by the value of the sample bias voltage. The vertical arrows show the "A" peak maxima - dependent on the in-plane electric field. The dashed line shows position of $1 s-2 p_{+}$transition peaks due to intra donor transition in the semi-insulating GaAs substrate. The oval shows the region of spectra where "B" peak arises for higher bias voltages. The inset shows the scheme of in-plane biasing of the sample: gray — active region of the quantum wells, black — two electrical contacts, $R_{\mathrm{L}}$ - load resistor enabling photocurrent monitoring. The magnetic field was applied perpendicularly to the quantum well plane.

the multiple quantum wells samples, the deeper lying wells are less influenced by the electric field of charged surface state due to the bigger distance and screening of upper QWs. Therefore we expect much stronger influence of the external bias voltage on the local electric field in the vicinity of shallow donor centers than in the case of single QW.

In the interpretation of experiments with single QW, the characteristic shape of obtained peak in $\mathrm{PC}(B)$ spectra was a result of an interplay of the following mechanisms [5]: (i) increasing population of neutral donors due to the magnetic field induced electron localization, (ii) damping of the $\mathrm{PC}(B)$ signal connected with changes of the initial state symmetry of $1 s-2 p_{+1}$ transition, which decreases the value of a proper dipole matrix element, (iii) changes in the density of states of shallow donors. The crucial role in this explanation is attributed to the existence 
of in-plane fluctuations of the local electric potential characterized by a certain distribution of their amplitude and spatial extent. These parameters determine the shape of $\mathrm{PC}(B)$ spectra and its evolution during changes of the external bias voltage. This interpretation assumes that the resultant shape of the $\mathrm{PC}(B)$ spectra originates totally from the first step of the signal generation process, i.e., absorption of the FIR photon. The second step, i.e. a phonon assisted transfer of electron into the conduction band remains unchanged by the external electric fields since they do not change the efficiency of this process.

The complementary approach is to consider the opposite situation. The first step remains unaffected by external fields. Due to the continuous character of the density of available donor transition states, there are always donors which can absorb a FIR photon and only changes in the second step of the $\mathrm{PC}(B)$ signal generation are influenced by the external fields. The continuum of available donor states is due to the existence of donors located at every possible position relative to the QW center. This, in turn, causes the characteristic energies of shallow donor: binding energy and transitions energies, to be different for each donor location $[7,8]$. The crossed electric and magnetic fields configuration causes the relaxation of the selection rules for optical absorption and therefore one obtains as a result a more uniform density of transition energies available for electrons absorbing photons. Therefore, the first step of the electron transfer into the conduction band can be assumed to be always the same, regardless the applied external fields. All observed changes due to external fields take place because of modification in the efficiency of phonon assisted electron transfer into the conduction band.

In the multiple QW structure investigated, one expects that the variation of the external bias, giving the in-plane external electric field $E_{\text {ext }}$, causes significant changes of the resultant electric field $E_{\text {eff }}$, which is the vector sum of $E_{\text {ext }}$ and electric field built in the structure. Detailed calculations show that the built-in electric field in the structure is perpendicular to the QW plane and is equal to $\approx 300 \mathrm{~V} / \mathrm{cm}$ for the first quantum well and equal to $\approx 10 \mathrm{~V} / \mathrm{cm}$ for the 15 th quantum well from the top surface. The fluctuations of local electric potential, which were previously mentioned, influence this electric field in the way of changing its value and also its direction - causing deviations from the perpendicular direction and thus giving the different orientations of $E_{\text {eff }}$ in each donor location in each QW. By applying the external magnetic field we reach the configuration of crossed electric and magnetic field $B$, but in each QW and at each donor place in the structure one can imagine a slightly different angle between $E_{\text {eff }}$ and $B$. It is easy to understand that the external bias, giving the horizontal electric field of the order of $10-50 \mathrm{~V} / \mathrm{cm}$, influences mainly the deeper QWs $(\approx 15 \mathrm{th})$. There are works [9] describing the statistical approach to the ladder of excited states of the hydrogen atom subjected to the external electric field $E_{\text {eff }}$ and magnetic field $B$. It is shown that the distribution of excited states as a function of their energy spacing can be described by the Brody function [10] characterized by the parameter $q$, where 


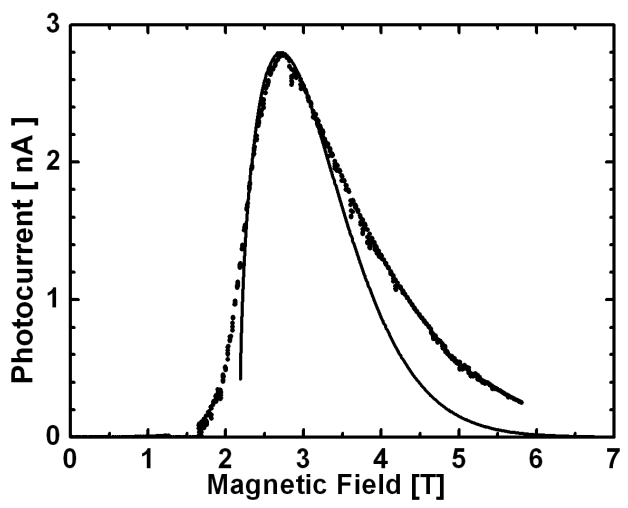

Fig. 2. The example of experimental data (points) fitted with the Brody function (solid line) for the following parameters: the angle between $E_{\text {eff }}$ and $B$ equal to $65^{\circ}$ and the Brody function parameter $q=0.5$. The experimental data were taken for bias voltage $U_{\mathrm{b}}=8 \mathrm{~V}$ after having subtracted the background signal, which was phenomenologically modeled on the base of all recorded spectra.

$q \in\langle 0 ; 1\rangle$. This function is an interpolation between the Poisson $(q=0)$ and the Wigner $(q=1)$ distribution of energy levels. The remaining parameters of the Brody distribution are: (i) the angle $\beta$ between $E_{\text {eff }}$ and $B$, (ii) a mean spacing between energy levels and (iii) a relative value of $E_{\text {eff }} / B^{\kappa}$, where $\kappa$ is a scaling parameter $[11,12]$. We have fitted the Brody function to one of experimental spectra in order to show the possibility of getting a reasonable agreement in the shape of observed and fitted peaks. The result is shown in Fig. 2. We assumed the energy spacing (on the Brody distribution graph) to be proportional to the magnetic field. The numerical fit of the Brody function has been done for the following parameters: the angle between $E_{\text {eff }}$ and $B, \beta \approx 65^{\circ}$ and for the parameter $q=0.5$. It is visible that the contribution of a single $\mathrm{QW}$ is already a quite well fit to the experimental data.

This result shows that by applying a statistical description of excited shallow donor states in our system one can expect to describe the evolution of the ladder of excited levels of shallow donors in QW with changing external fields in the language of these parameters. This evolution, in turn, influences the shape and changes of $\mathrm{PC}(B)$ spectra. Figure 2 shows the contribution of only one QW. Taking into account the whole set of QWs would give a much better correspondence to the experimental data.

In the considered structure of multiple quantum wells the observed peaks result probably from processes which are a sum of both presented approaches. The upper QWs contribute to the measured signal mainly according to the first mechanism presented while the deeper QWs - mainly to the second one. It is shown that considering even the single approach gives a good agreement with the experimental data. 


\section{Acknowledgments}

This work was partially supported by the MTKD-CT-2005-029671 grant.

\section{References}

[1] M. Szot, K. Karpierz, J. Kossut, M. Grynberg, in: Proc. Conf. Phys. of Semicond. ICPS-27, 27th Int. Conf. Phys. Semicond., Eds. J. Menendez, C.G. Van de Walle, AIP Publishing, Melville, New York 2005, p. 1129.

[2] K. Karpierz, M. Szot, J. Łusakowski, M. Grynberg, Acta Phys. Pol. A 112, 237 (2007).

[3] P.D. Wang, S.N. Holmes, Tan Le, R.A. Stradling, T. Fergusson, A.G. de Oliveira, Semicond. Sci. Technol. 7, 767 (1992).

[4] D.M. Larsen, Phys. Rev. B 8, 535 (1973).

[5] K. Karpierz, M. Szot, J. Łusakowski, M. Grynberg, to be published in J. Phys.: Condens. Matter.

[6] Sh.M. Kogan, T.M. Lifshitz, Phys. Status Solidi A 39, 11 (1977).

[7] G. Bastard, Phys. Rev. B 24, 4714 (1981).

[8] R.L. Green, K.K. Bajaj, Phys. Rev. B 34, 951 (1986).

[9] J. Main, M. Schwacke, G. Wunner, Phys. Rev. A 57, 1149 (1998).

[10] T.A. Brody, J. Flores, J.B. French, P.A. Mello, A. Pandey, S.S.M. Wong, Rev. Mod. Phys. 53, 385 (1981).

[11] T. Bartsch, J. Main, G. Wunner, Phys. Rev. A 67, 063411 (2003).

[12] G. Wiebusch, J. Main, K. Krüger, H. Rottke, A. Holle, K.H. Welge, Phys. Rev. Lett. 62, 2821 (1989). 